\title{
柔性可穿戴电子传感器研究进展
}

\author{
钱金金 $a, b$ 苏萌 $a, b$ 李风煜*, $a$ 宋延林*, \\ ( ${ }^{a}$ 中国科学院化学研究所中国科学院绿色印刷重点实验室 北京 100190) \\ ( ${ }^{b}$ 中国科学院大学化学与化工学院 北京 100049)
}

\begin{abstract}
摘要 随着智能终端的普及, 可穿戴电子设备呈现出巨大的市场前景. 传感器作为核心部件之一, 将影响可穿戴设备 的功能设计与未来发展. 柔性可穿戴电子传感器具有轻薄便携、电学性能优异和集成度高等特点, 使其成为最受关注 的电学传感器之一. 综述了近年来柔性可穿戴电子传感器的研究进展, 包括压阻、电容、压电、力致发光和摩擦电等 信号转换机理，金属、无机半导体、有机和碳材料等柔性可穿戴电子的常用材料, 柔性电子传感器的印刷制造及其在 体温和脉搏检测、表情识别和运动监测等方面的最新应用, 最后提出了柔性可穿戴电子传感器面临的挑战与未来发展 方向.
\end{abstract}

关键词＼cjkstart可穿戴电子; 柔性传感器; 印刷制造; 人体监测

\section{Research Progress in Flexible Wearable Electronic Sensors}

$$
\text { Qian, Xin }{ }^{a, b} \text { Su, Meng }{ }^{a, b} \quad \text { Li, Fengyu* }{ }^{*, a} \quad \text { Song, Yanlin*,a }
$$

( ${ }^{a}$ Key Laboratory of Green Printing, Institute of Chemistry, Chinese Academy of Sciences, Beijing 100190)

( ${ }^{b}$ School of Chemistry and Chemical Engineering, University of Chinese Academy of Sciences, Beijing 100049)

\begin{abstract}
With the development of intelligent terminals, wearable electronic devices show a great market prospect. As one core component of the wearable electronic device, the sensor will exert a significant influence on the design and function of the wearable electronic device in the future. Compared with the traditional electrical sensors, flexible wearable sensors have the advantages of being light, thin, portable, highly integrated and electrically excellent. It has become one of the most popular electronic sensors. This review focused on recent research advances of flexible wearable sensors, including signal transduction mechanisms, general materials, manufacture processes and recent applications. Piezoresistivity, capacitance and piezoelectricity are three traditional signal transduction mechanism. For accessing the dynamic pressure in real time and developing stretchable energy harvesting devices, sensors based on the mechanoluminescent mechanism and triboelectric mechanism are promising. Common materials used in flexible wearable electronic sensors, such as flexible substrates, metals, inorganic semiconductors, organics and carbons, are also introduced. In addition to the continuously mapping function, wearable sensors also have the practical and potential applications, which focused on the temperature and pulse detection, the facial expression recognition and the motion monitoring. Finally, the challenges and future development of flexible wearable sensors are presented.
\end{abstract}

Keywords wearable electronics; flexible sensor; printing manufacture; body monitoring

\section{1 引言}

传感器在人体健康监测方面发挥着至关重要的作 用 ${ }^{[1]}$. 近年来, 人们已经在可穿戴可植入传感器领域取 得了显著进步, 例如利用电子皮肤向大脑传递皮肤触觉 信息 ${ }^{[2]}$, 利用三维微电极实现大脑皮层控制假肢 ${ }^{[3]}$, 利 用人工耳蜗恢复病人听力等 ${ }^{[4]}$. 然而, 实现柔性可穿戴 电子传感器的高分辨、高灵敏、快速响应、低成本制造 和复杂信号检测仍然是一个很大的挑战 ${ }^{[5]}$. 本文综述了 近年来柔性可穿戴电子传感器的研究进展, 包括压阻、 电容、压电、力致发光和摩擦电五种信号转换机制, 金
属、无机半导体、有机和碳材料等适用于柔性可穿戴电 子的材料, 柔性电子传感器的印刷制造及其在体温和脉 搏检测、表情识别及运动监测等方面的最新应用.

\section{2 柔性可穿戴电子传感器机械力信号转换}

如何有效地将外部刺激转化为电信号是柔性可穿 戴电子传感器监测身体健康状况的关键技术. 柔性可穿 戴电子传感器的信号转换机制主要分为压阻 ${ }^{[6]}$ 、电容 ${ }^{[7]}$ 和压电 ${ }^{[8]}$ 三大部分. 近年来为了实时扫描动态压力和发 展可拉伸的能量收集器件, 基于力致发光 ${ }^{[9]}$ 、摩擦发

\footnotetext{
* E-mail: forrest@iccas.ac.cn \& ylsong@iccas.ac.cn

Received April 6, 2016; published May 26, 2016.

Project supported by the 973 Program (No. 2013CB933004), the National Natural Science Foundation of China (Grant Nos. 51203166, 51473172, 51473173, 21203209, 21301180 and 21303218) and the "Strategic Priority Research Program" of Chinese Academy of Sciences (Grant No. XDA09020000).

项目受 973 计划(No. 2013CB933004)、国家自然科学基金(Grant Nos. 51203166, 51473172, 51473173, 21203209, 21301180 and 21303218)和中科院战略 性先导科技专项(Grant No. XDA09020000).
} 
电 $^{[10]}$ 机制的传感器受到了高度关注(见图 1).

\section{1 压阻}

压阻传感器可以将外力转换成电阻的变化, 进而可 以方便地用电学测试系统间接探测外力变化. 而导电物 质间导电路径的变化是获得压阻传感信号的常见机 理 ${ }^{[11]}$. 由于其简单的设备和信号读出机制, 这类传感器 得到广泛应用.

压阻传感器导电物质和导电弹性复合材料之间接 触电阻的变化与施加压力的平方根成正比 ${ }^{[12]}$, 因此可 以探测到微小的压力, 拓展检测范围. 程文龙等 ${ }^{[13]}$ 发展 了一种简单实用的高灵敏压阻传感器, 其在弹性基底上 构筑了金纳米线薄层和电极阵列. 这种器件具有 $13 \sim$ $50000 \mathrm{~Pa}$ 宽的检测范围. 为了增强灵敏性, 实现对接触 力的扫描, 鲍哲楠等 ${ }^{[2]}$ 利用具有雉状微结构的压阻传感 器制备了一种可以向大脑传递触觉信息的电子皮肤.

通常实现压阻传感器高灵敏性, 需要以高电阻为代 价. 最近, 张广宇等 ${ }^{[14]}$ 开发了一种纳米石墨烯的压阻薄 膜，灵敏度提高的同时，降低了功耗.

为了实现差异化的电阻响应, 我们 ${ }^{[15]}$ 首次采用硅 柱诱导的印刷方法简便组装了振幅和周期可调的微米 级曲线阵列压阻传感器. 其在 1000 次 5\%的拉伸-舒张 循环下保持了稳定的实时响应. 实验中成功组装了银纳 米粒子和导电聚合物. 由于基于硅柱间液桥收缩和纳米 颗粒自组装的原理，使这种方法具有普适性.

\section{2 电容}

电容是衡量平行板间容纳电荷能力的物理量. 传统 的电容传感器通过改变正对面积 $S$ 和平行板间距 $d$ 来探 测不同的力, 例如压力, 剪切力等 ${ }^{[16]}$.

电容式传感器的主要优势在于其对力的敏感性强, 可以实现低能耗检测微小的静态力 ${ }^{[17]}$. 鲍哲楠等 ${ }^{[18]}$ 在 弹性基底上制备了电容型透明可拉伸的碳纳米管传感 器, 对压力和拉力同时有响应.

\section{3 压电}

压电材料是指在机械压力下可以产生电荷的特殊 材料. 这种压电特性是由存在的电偶极矩导致的. 电偶 极矩的获得是靠取向的非中心对称晶体结构变形 ${ }^{[19]}$, 或者孔中持续存在电荷的多孔驻极体 ${ }^{[20]}$. 压电系数是 衡量压电材料能量转换效率的物理量, 压电系数越高, 能量转换的效率就越高. 高灵敏, 快速响应和高压电系 数的压电材料被广泛应用于将压力转换为电信号的传 感器.

压电无机物是典型的高压电系数，低柔性的材料; 而压电聚合物正好相反. 为了探索高压电系数的柔性压 力传感器, 人们尝试了一系列方法, 包括在柔性基底上 构筑压电无机薄膜 ${ }^{[21]}$ ，使用压电聚合物或无机/聚合物 复合物 ${ }^{[22]}$ 和构筑稳定的压电驻极体 ${ }^{[23]}$. 最近, 具有良好 压电特性和机械稳定性的纳米线和纳米带吸引了国际 上对集成高分辨感知阵列传感器的浓厚兴趣 ${ }^{[8]}$.

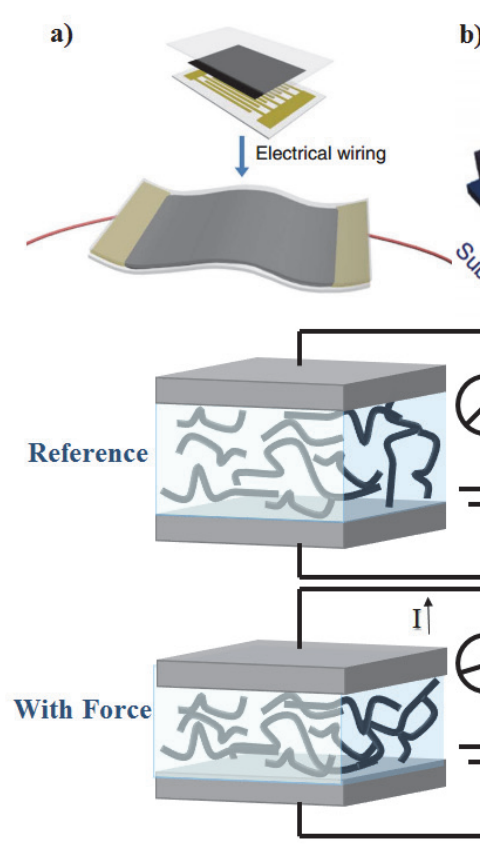

Piezoresistivity

b)
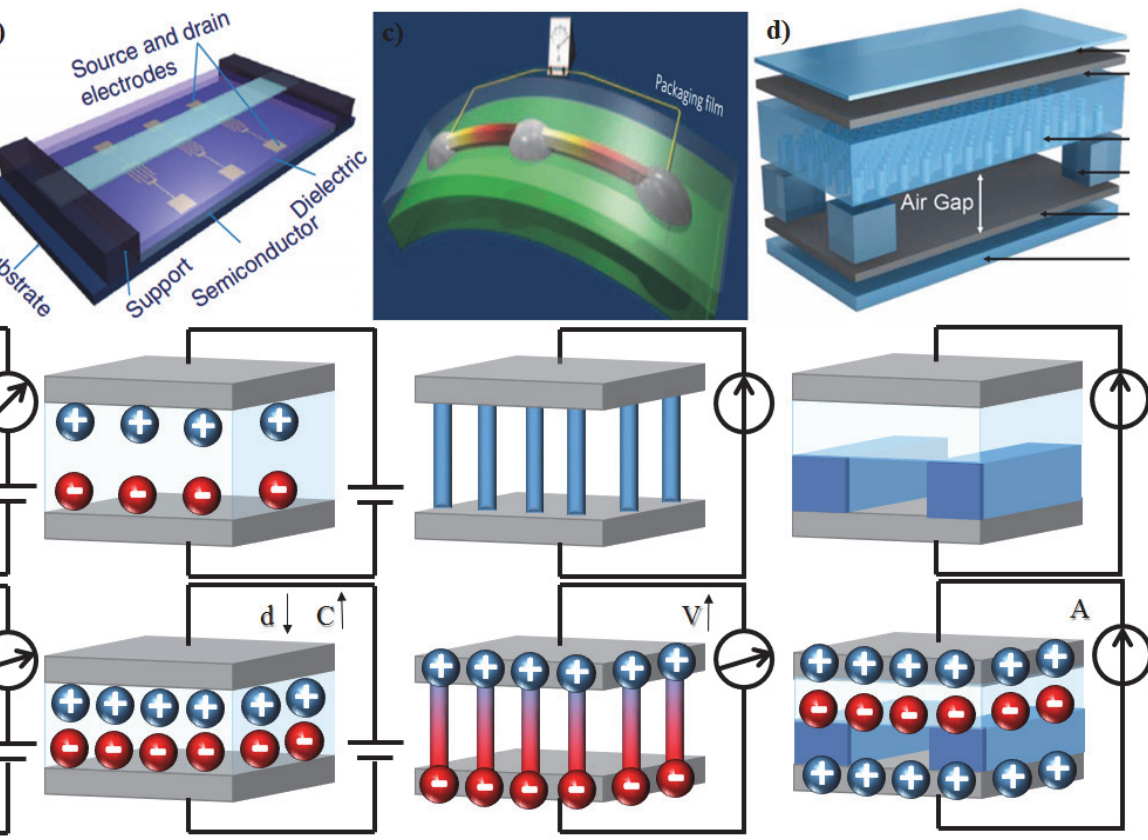

Piezoelectricity

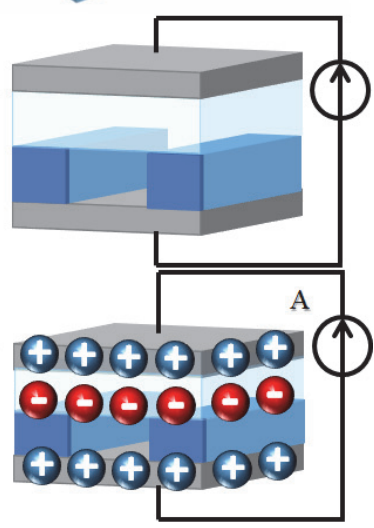

Triboelectricity

图 1 柔性可穿戴电子传感器四种信号传导机制和器件的示意图

Figure 1 Schematic illustrations of four common transduction methods and representative devices

(a) piezoresistivity, (b) capacitance, (c) piezoelectricity and (d) triboelectricity 


\section{4 其他信号转换机制}

除了上述提到的方法外, 其他新颖的信号转换机制 发展也促进了柔性可穿戴电子传感器的广泛应用. 不同 于传统静态力传感器, 力致发光传感器可以实现动态力 的实时显示 ${ }^{[24]}$. 另一种基于接触电和静电感应的摩擦 电传感器可以自驱动地监测触觉, 推动了无需外部能源 供给的自驱动传感器向前发展 ${ }^{[10]}$.

\section{3 柔性可穿戴电子的常用材料}

\section{1 柔性基底}

为了满足柔性电子器件的要求, 轻薄、透明、柔性 和拉伸性好、绝缘耐腐蚀等性质成为了柔性基底的关键 指标. 在众多柔性基底的选择中, 聚二甲基硅氧烷 (PDMS)成为了人们的首选. 它的优势包括方便易得、化 学性质稳定、透明和热稳定性好等. 尤其在紫外光下粘 附区和非粘附区分明的特性使其表面可以很容易的粘 附电子材料 ${ }^{[25]}$.

很多柔性电子设备通过降低基底的厚度来获得显 著的弯曲性; 然而, 这种方法局限于近乎平整的基底表 面 ${ }^{[26]}$. 相比之下，可拉伸的电子设备可以完全粘附在复 杂和凹凸不平的表面上.

目前, 通常有两种策略来实现可穿戴传感器的拉伸
性(见图 2). 第一种方法是在柔性基底上直接键合低杨 氏模量的薄导电材料. 第二种方法是使用本身可拉伸的 导体组装器件. 通常是由导电物质混合到弹性基体中制 备 ${ }^{[27]}$. Someya 等 ${ }^{[28]}$ 制备了可拉伸的有机发光二极管有 源矩阵. 含氟共聚物的高弹膜中均匀分散着可印刷的弹 性导体, 如单壁碳纳米管. 用离子液体法制备的细长碳 纳米管，其拉伸性高达 $100 \%$, 导电性高达 $100 \mathrm{~S} \bullet \mathrm{cm}^{-1}$.

几何图案和器件设计方面, 网状结构被用来进一步 增强拉伸性和适应性 ${ }^{[29]}$. Rogers 等 ${ }^{[30]}$ 首先提出把电学性 能优异的刚性传统无机材料粘附在弹性基底表面. 将无 机半导体(包括电子元件和连接电路)组装在可拉伸的器 件上. 与众不同的是, 高杨氏模量机械平面层的张力是 可以忽略的，而复杂的波浪结构吸收了基底压缩-舒张 过程中产生的大部分拉伸应变 ${ }^{[31]}$. 这种岛一桥设计首次 显著提高了传感器的可拉伸性; 这种设计中, 刚性大的 活动模块作为浮动的岛屿, 刚性小的连线充当拉桥 ${ }^{[32]}$. 可变形连接部分的非共面结构, 包括直带和蛇纹, 可以 让传感器经历复杂的形变, 比如旋转和扭曲. 最近, 自 相似的分形层是连线的选择之一, 这种分形导线有利于 适应不同的形变 ${ }^{[33]}$. 为了适应实际的需要, 在连线和软 基底之间的三种接触模式得到有效利用，包括非键、部 分键合与完全键合. 举例来说，非键设计形变自由，并 a)

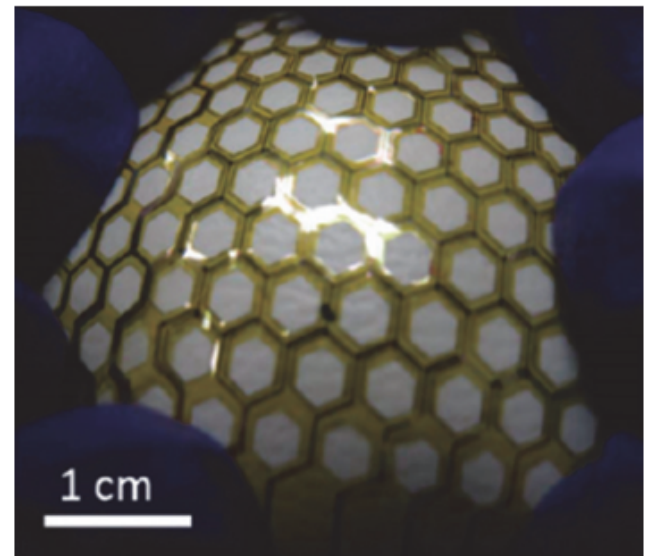

c)
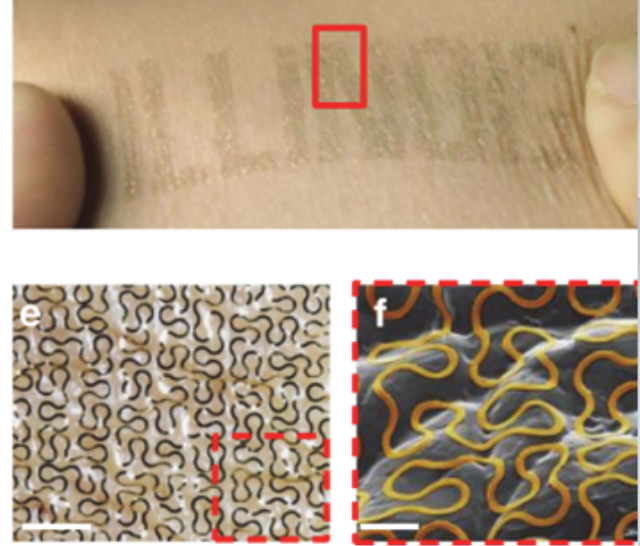

b) fabr. ultrathin circuit mesh

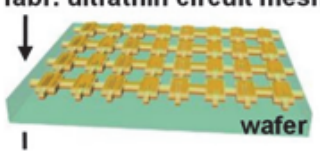
liftoff; deposit $\mathrm{Cr} / \mathrm{SiO}_{2}$ $\downarrow$

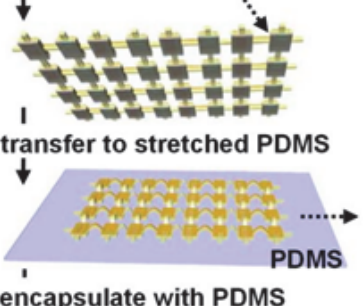
encapsulate with PDMS d)
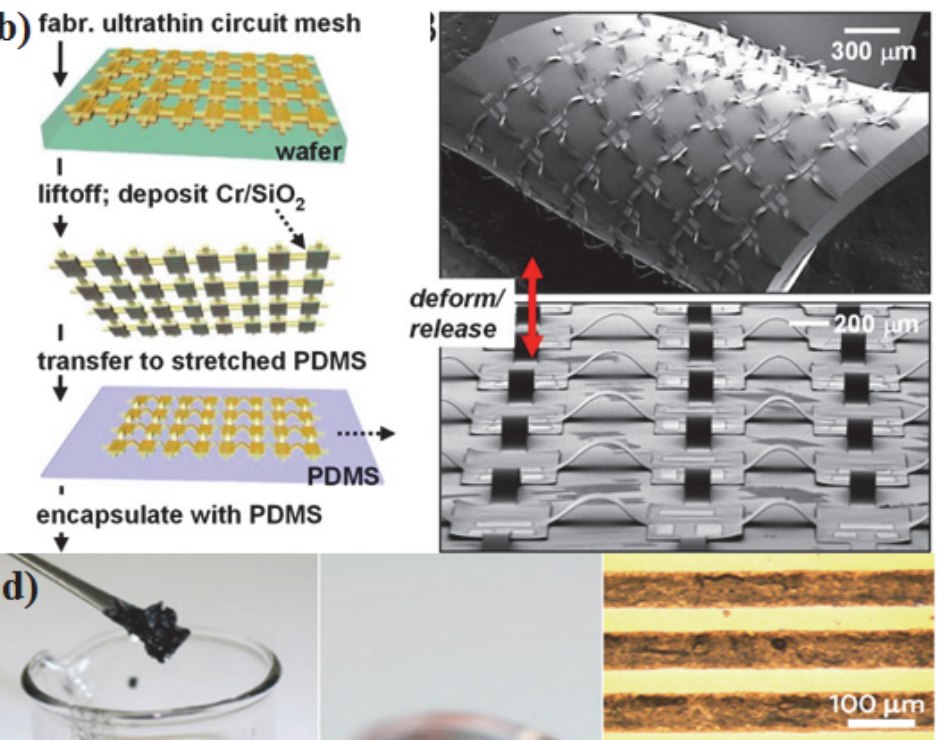

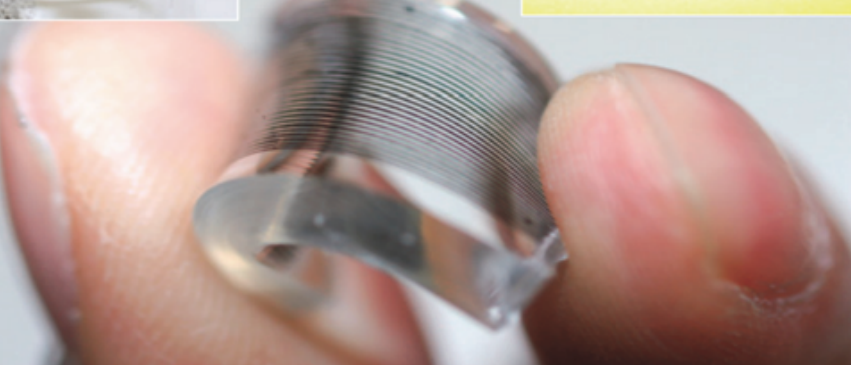

图 2 实现可拉伸性的不同策略

Figure 2 Different strategies to achieve stretchability

Geometric pattern design: (a) Net-shaped structural design. (b) Noncoplanar mesh design. (c) Fractal design. (d) Use of elastic conductors 
且连线的暴露对物理损伤更加敏感, 使得其表现了最好 的机械性能.

\section{2 金属材料}

金属材料一般为金银铜等导体材料, 主要用于电极 和导线. 对于现代印刷工艺而言, 导电材料多选用导电 纳米油墨, 包括纳米颗粒和纳米线等 ${ }^{[34]}$. 金属的纳米粒 子除了具有良好的导电性外, 还可以烧结成薄膜或导 线. Park 等 ${ }^{[35]}$ 发展了一种电路, 通过静电纺丝技术大规 模生产银纳米颗粒覆盖的橡胶纤维的电路. 在 $100 \%$ 拉 力下, 导电性达到 $2200 \mathrm{~S} \cdot \mathrm{cm}^{-1}$.

\section{3 无机半导体材料}

以 $\mathrm{ZnO}$ 和 $\mathrm{ZnS}$ 为代表的无机半导体材料由于其出 色的压电特性, 在可穿戴柔性电子传感器领域显示出了 广阔的应用前景 ${ }^{[7]}$. 一种基于直接将机械能转换为光学 信号的柔性压力传感器被开发出来 ${ }^{[36]}$ (见图 3). 这种矩 阵利用了 $\mathrm{ZnS}: \mathrm{Mn}$ 颗粒的力致发光性质. 力致发光的核 心是压电效应引发的光子发射. 压电 $\mathrm{ZnS}$ 的电子能带在 压力作用下产生压伏效应而产生倾斜, 这样可以促进
$\mathrm{Mn}^{2+}$ 的激发，接下来的去激发过程发射出黄光(580 nm 左右)。一种快速响应(响应时间小于 $10 \mathrm{~ms}$ )的传感器就 是由这种力致发光转换过程所得到, 通过自上而下的光 刻工艺，其空间分辨率可达 $100 \mu \mathrm{m}$. 这种传感器可以记 录单点滑移的动态压力, 其可以用于辨别签名者笔迹和 通过实时获得发射强度曲线来扫描二维平面压力分布. 所有的这些特点使得无机半导体材料成为未来快速响 应和高分辨压力传感器材料领域最有潜力的候选者之

\section{4 有机材料}

大规模压力传感器阵列对未来可穿戴传感器的发 展非常重要 ${ }^{[37]}$. 基于压阻和电容信号机制的压力传感 器存在信号串扰, 导致了测量的不准确, 这个问题成为 发展可穿戴传感器最大的挑战之一. 由于晶体管完美的 信号转换和放大性能, 晶体管的使用为减少信号串扰提 供了可能. 因此, 在可穿戴传感器和人工智能领域的很 多研究都是围绕如何获得大规模柔性压敏晶体管展开 的. a)

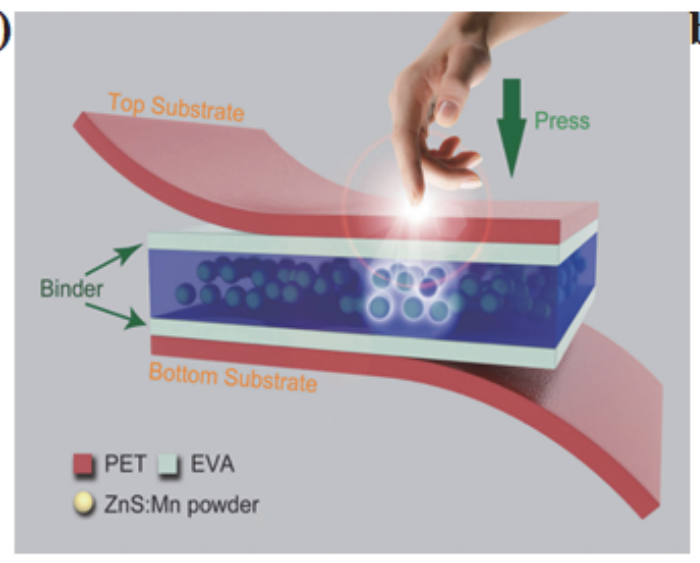

d)

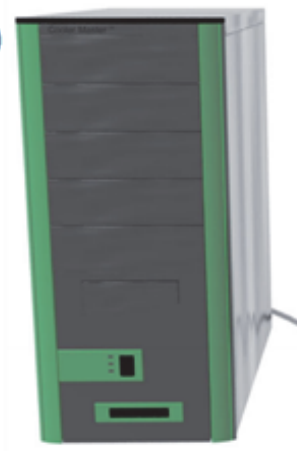

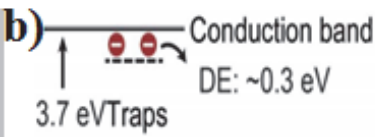

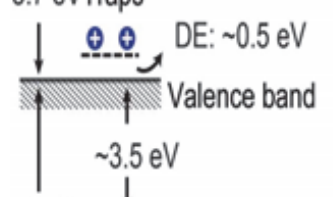

c)

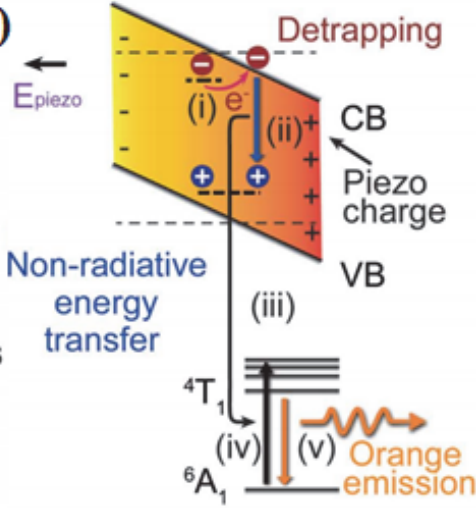

Stress applied
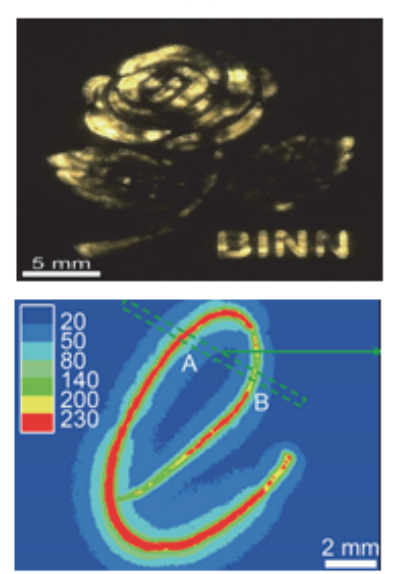

图 3 基于力致发光的压力扫描

Figure 3 Pressure mapping based on piezophotonics

(a) Schematic structure of the device. (b) Band diagram of ZnS:Mn, which indicates the action mechanism of piezophotonic effect. (c) Dynamic pressure mapping of 2D planar and single-point models. (d) Signature recording and high-resolution pressure mapping 
典型的场效应晶体管是由源极、漏极、栅极、介电 层和半导体层五部分构成. 根据多数载流子的类型可以 分为 $\mathrm{p}$ 型(空穴)场效应晶体管和 $\mathrm{n}$ 型(电子)场效应晶体 管. 传统上用于场效应晶体管研究的 $\mathrm{p}$ 型聚合物材料主 要是噻吩类聚合物, 其中最为成功的例子便是聚 (3-己 基噻吩)(P3HT)体系. 菜四酰亚二胺(NDI)和萠四酰亚二 胺(PDI)显示了良好的 $\mathrm{n}$ 型场效应性能, 是研究最为广泛 的 $\mathrm{n}$ 型半导体材料, 被广泛应用于小分子 $\mathrm{n}$ 型场效应晶 体管当中 ${ }^{[38]}$. 通常晶体管参数有载流子迁移率、运行电 压和开/关电流比等. 与无机半导体结构相比, 有机场效 应晶体管(OFET) 具有柔性高和制备成本低的优点, 但 也有载流子迁移率低和操作电压大的缺点. 近来, 鲍哲 楠等 ${ }^{[39]}$ 设计了一种具有更高噪声限度的逻辑电路. 通 过优化掺杂厚度或浓度, 基于 $\mathrm{n}$ 型和 $\mathrm{p}$ 型碳纳米管晶体 管的设计可用来调节阈值电压.

晶体管出色的电学开关行为引起了科学家对压力 传感器的广泛兴趣. Someya 等 ${ }^{[40]}$ 首次使用堆叠了压敏 橡胶的有机场效应晶体管矩阵来作为高性能压力传感 阵列. 晶体管用来低能耗快速表达, 它的栅极和漏极分 别连接字线和位线, 源极通过压敏的弹性橡胶接地. 压 敏橡胶网格的电阻用作感知压力的变化, 晶体管㮽电压 的变化, 导致漏极电流的变化.

为了满足更多的应用, 人们亟需发展一种检测压力 范围广, 响应速度快的矩阵策略. 鲍哲楠等 ${ }^{[41]}$ 在硅片上 集成了一种新型高压敏感的有机晶体管, 其具有微结构 的可压缩栅电介质(见图 4). 相比于无结构或其他微结 构的膜, 具有雉状结构的 PDMS 层电容式传感器极大地 提高了压力敏感性. 原因是 PDMS 层和有机半导体间空 隙的提高使得介电常数降低. 在此基础上, 进一步在塑 料基底上发展了柔性的压敏矩阵. 这种基于微结构橡胶 的矩阵具有反应迅速和高压敏感性的特点, 其可以精确 的扫描静态压力分布和监测健康 ${ }^{[42]}$. 尽管如此, 该类器 件还是存在介电层的弹性极限问题, 超高灵敏度压力传 感器件 $\left(\geqslant 100 \mathrm{kPa}^{-1}\right)$ 难以实现. 朱道本等 ${ }^{[43]}$ 首次成功构 建了柔性悬浮栅有机薄膜晶体管(SGOTFT), 有效避免 了介电层弹性极限问题并使得器件的压力传感特性取 决于栅极的机械性质. 基于该原理, 科研人员构建了灵 敏度高达 $192 \mathrm{kPa}^{-1}$ 的超高灵敏度压力传感器. 此外, 该 类器件展现了非常优异的柔韧性、稳定性和低电压操作 特性, 相应的器件阵列成功应用于人体脉搏的检测和微 小物体的运动追踪, 在人工智能和可穿戴健康监测方面 显示了非常好的应用前景.

\section{5 碳材料}

柔性可穿戴电子传感器常用的碳材料有碳纳米管 和石墨烯等. 碳纳米管具有结晶度高、导电性好、比表 面积大、微孔大小可通过合成工艺加以控制, 比表面利 用率可达 $100 \%$ 的特点. 石墨烯具有轻薄透明, 导电导 热性好等特点. 在传感技术、移动通讯、信息技术和电
动汽车等方面具有极其重要和广阔的应用前景.
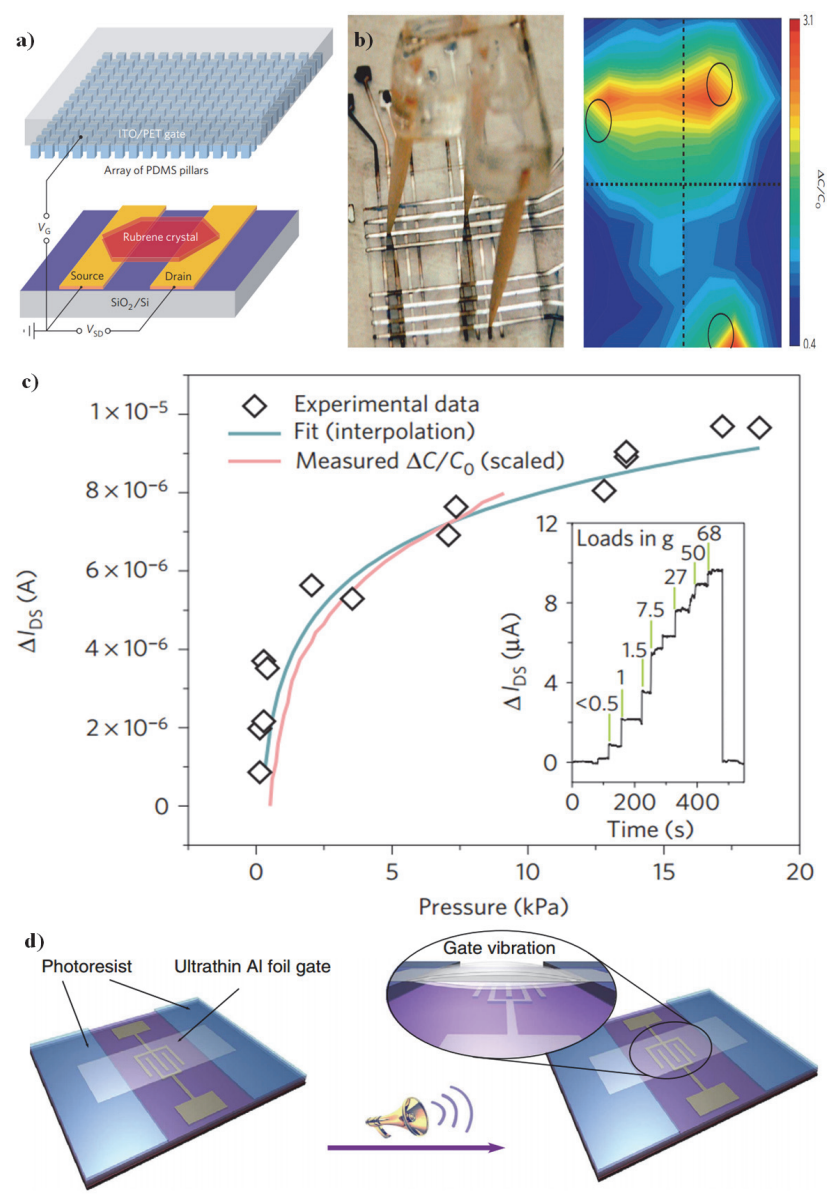

图 4 基于栍介电层几何设计的高灵敏的矩阵传感器

Figure 4 Highly sensitive active matrix sensors with geometric design of gate dielectric

(a) Pressure-sensitive OFET with microstructured PDMS dielectric layer. (b) Flexible pixel-type capacitive pressure sensor array using a microstructured PDMS film as a dielectric layer. (c) Change in $I_{\mathrm{DS}}$ of the OFET in response to pressure, which is proportional to the change in relative capacitive and exhibits a rapid response. (d) Device geometry for ultra-sensitive detection of acoustic wave

在碳纳米管的应用上, Chun 等 ${ }^{[44]}$ 利用多臂碳纳米 管和银复合并通过印刷方式得到的导电聚合物传感器, 在 $140 \%$ 的拉伸下, 导电性仍然高达 $20 \mathrm{~S} \cdot \mathrm{cm}^{-1}$. 在碳纳 米管和石墨烯的综合应用上，Lee 等 ${ }^{[45]}$ 制备了可以高度 拉伸的透明场效应晶体管，其结合了石墨烯/单壁碳纳 米管电极和具有褶皱的无机介电层单壁碳纳米管网格 通道. 由于存在褶皱的氧化铝介电层, 在超过一千次 $20 \%$ 幅度的拉伸一舒张循环下，没有漏极电流变化，显 示出了很好的可持续性.

\section{4 柔性电子传感器的印刷制造}

柔性传感器是可穿戴设备的关键部件之一, 以硅材 料为代表的传统半导体器件由于加工工艺复杂、设备投 入成本高、环境污染大以及芯片无法弯曲等内在局限性, 限制其在可穿戴设备领域的应用 ${ }^{[46]}$. 与传统自上而下 的光刻技术相比, 印刷电子技术拥有弯曲与拉伸性好、 
可以在柔性基底大规模制备、加工设备简单、成本低和 污染小等优点 ${ }^{[47]}$. 正因如此, 基于有机半导体材料和纳 米材料等的印刷电子技术在近年得到迅猛发展 ${ }^{[48]}$.

我们 ${ }^{[49]}$ 在功能纳米材料的可控组装、精细图案化技 术以及印刷电子应用方面开展了一系列广泛而深入的 研究(见图 5). 我们通过调控墨水、基材等打印条件, 成 功制备了一系列特殊结构和图案: 利用 “咖啡环” 现象 制备线宽可达 $5 \mu \mathrm{m}$ 的金属纳米粒子图案 ${ }^{[50]}$; 提出了一 种通过控制液膜破裂实现了多种纳米粒子大面积精确 组装的普适方法, 这种新型图案化技术可以简便地进行 纳米粒子微、纳米尺度图案的精确组装, 可以通过 “印 刷” 方式大面积制备纳米粒子组装的精细图案和功能器 件, 乃至实现单个纳米粒子的组装与图案化 ${ }^{[51]}$; 通过喷 墨打印技术构筑微米尺度的电极图案作为 “模板” ，控 制纳米材料的组装过程成功制备了最高精度可达 $30 \mathrm{~nm}$ 的图案, 并实现了柔性电路的应用. 这种新型的图案化 技术非常简便地实现了功能纳米材料的微纳米精确图 案化组装, 在过程中完全避免了传统的光刻工艺, 这种 “全增材制造”的方法通过 “先打印, 再印刷” 的方式, 能
够大面积制备纳米材料组装的精细图案和功能器件 [52]; 利用特殊图案化硅柱阵列为模板制备了周期与振幅可 控的曲线阵列, 真空蒸镀上金电极, 得到对微小形变有 稳定电阻变化的传感器芯片 ${ }^{[15]}$.

\section{5 可穿戴传感器的应用}

可穿戴传感器除了具有压力传感功能, 还具有现实 和潜在应用的多种功能, 体温和脉搏检测、表情识别和 运动监测等.

\section{1 温度检测}

人体皮肤对温度的感知帮助人们维持体内外的热 量平衡 ${ }^{[53]}$. 电子皮肤的概念最早由 Rogers 等提出, 由多 功能二极管、无线功率线圈和射频发生器等部件组成. 这样的表皮电子对温度和热导率的变化非常敏感，可以 评价人体生理特征的变化, 比如皮肤含水量, 组织热导 率, 血流量状态和伤口修复过程 ${ }^{[54]}$.

为了提高空间分辨率、信噪比和响应速度, 有源矩 阵设计成为了最优选择之一. Ha 等 $^{[55]}$ 制备了包含单壁 a)
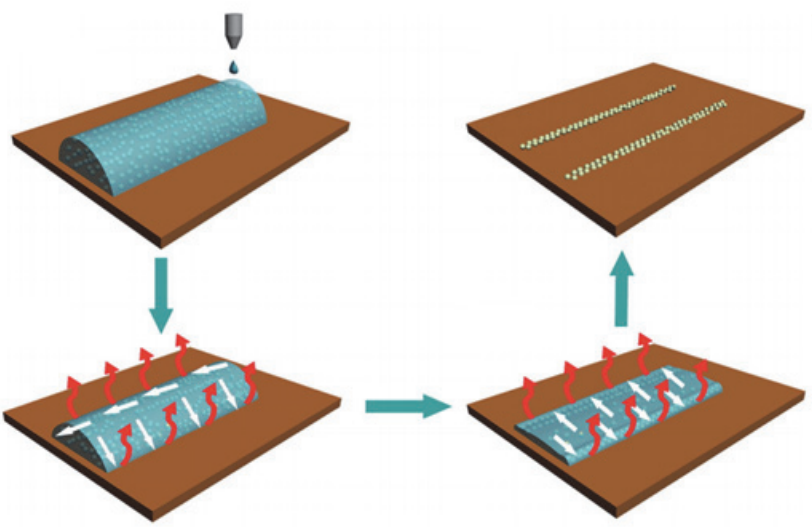

c)
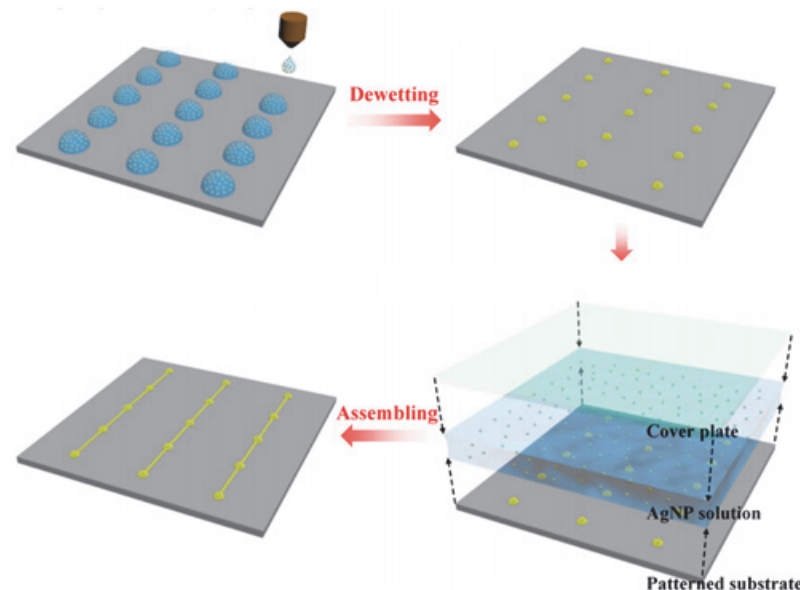

b)

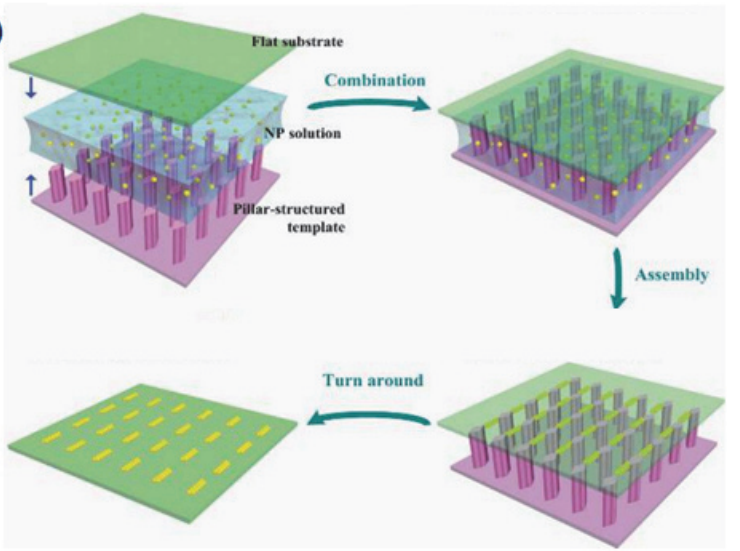

d)
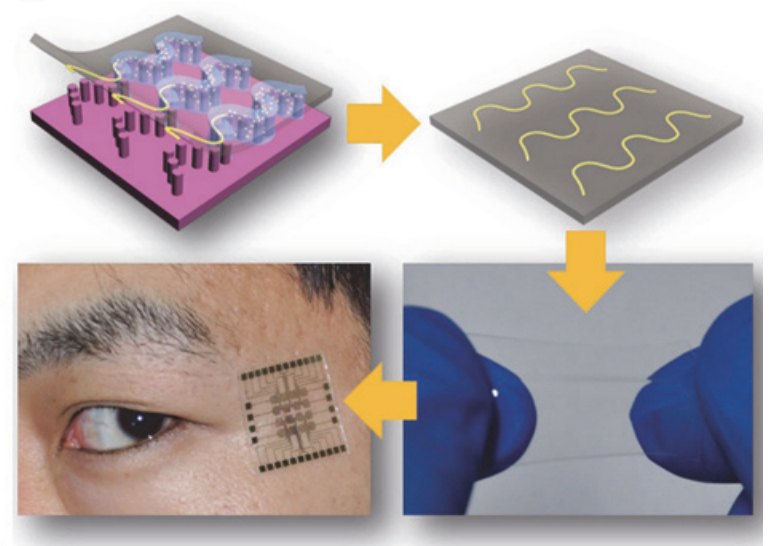

图 5 印刷电子的精细图案化控制

Figure 5 Fine patterning control of printing electronics

(a) Schematic illustration of inkjet printing of silver-nanoparticle patterns induced by the coffee-ring effect. (b) A general strategy to align a wide variety of NPs in one direction upon diverse substrates based on a sandwich-shaped assembly system. (c) A nonlithography strategy to fabricate nanoscale circuits by assembling conducting materials (e.g., AgNPs) on inkjet-printing patterned substrates through a space-confined assembly system. (d) The schematic illustration of the micro/nano curve array printed to flexible electronic devices and adopted to multianalysis for skin micromotion sensing 
碳纳米管薄膜晶体管的, 可拉伸的聚苯胺纳米纤维温度 传感器有源矩阵. 其展示了 $1.0 \% 0^{\circ} \mathrm{C}^{-1}$ 的高电阻灵敏性, 在 15 到 $45{ }^{\circ} \mathrm{C}$ 范围内得到了 $1.8 \mathrm{~s}$ 的响应时间, 在双向 拉伸 $30 \%$ 下依然保持稳定.

a)

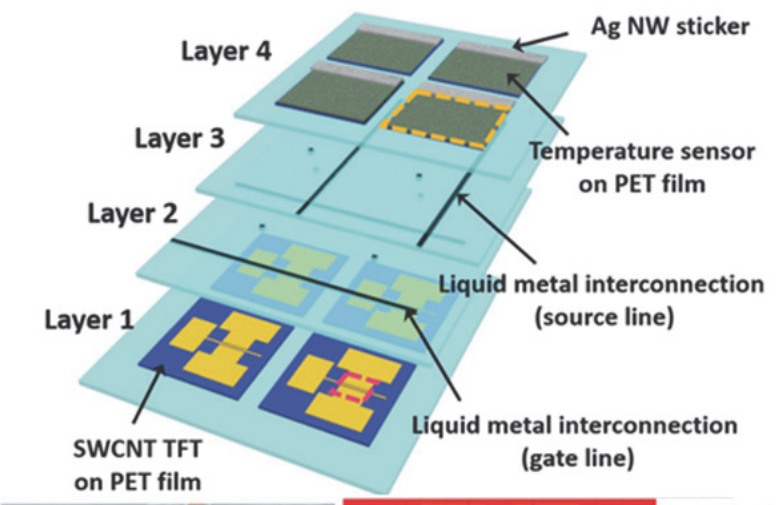

b)

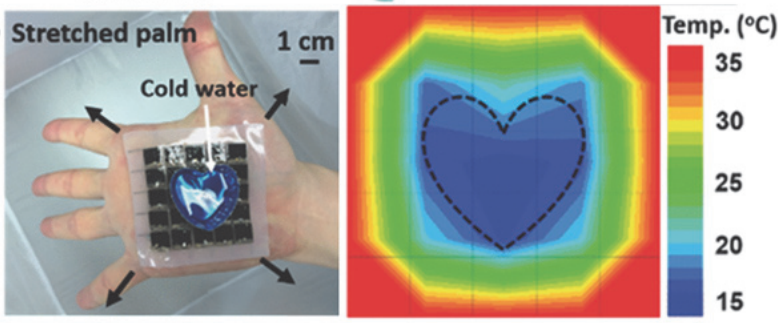

图 6 柔性电子传感器在温度监测上的应用

Figure 6 Flexible temperature-sensitive sensors

(a) Assembly of prepared layers, liquid metal injection, and formation of electrical contacts with the Ag NW sticker. (b) Optical images of the temperature array on the stretchable substrate attached onto the right palm where a heart-shaped cold water container $\left(\approx 15{ }^{\circ} \mathrm{C}\right)$ was positioned after stretching. The corresponding mapping of the temperature distribution via the measurement of the normalized drain current

\section{2 脉搏检测}

可穿戴个人健康监护系统被广泛认为是下一代健 康监护技术的核心解决方案. 监护设备不断地感知、获 取、分析和存储大量人体日常活动中的生理数据, 为人 体的健康状况提供必要的、准确的和长期的评估和反馈.

在脉搏监测领域, 可穿戴传感器具有以下应用优 势：(1) 在不影响人体运动状态的前提下长时间的采集 人体日常心电数据, 实时的传输至监护终端进行分析处 理; (2) 数据通过无线电波进行传输, 免除了复杂的连 线.

可以粘附在皮肤表面的电学矩阵在非植入健康监 测方面具有明显优势, 而且超轻超薄, 利于携带 ${ }^{[56]}$. 然 而, 人体具有很多非平整的表面和精细结构, 使得完全 贴附非常困难, 这是实时监测的挑战. 最近, 鲍哲楠 等 ${ }^{[57]}$ 发展了一种基于微毛结构的柔性压力传感器(见图 7). 这种传感器对信号的放大作用很强. 通过传感器与 不规则表皮的有效接触最大化，观察到了大约 12 倍的 信噪比增强. 另外, 这种 PDMS 的微毛结构表面层提供 了生物兼容性的非植入皮肤共形附着 ${ }^{[58]}$. 最后, 这种便 携式的传感器可以无线传输信号, 即使微弱的深层颈内
静脉搏动也可以获取到.

\section{3 表情识别}

统计发现，在人们的交流中通过语言传递的信息只 占约 7\%, 而大部分(约占 55\%)的信息靠表情传递 ${ }^{[59]}$. 随 着人机交互与情感计算技术的快速发展，人脸表情识别 已成为人们研究的热点. 目前, 基于图像采集的表情识 别技术存在五官捕捉缓慢、识别准确率低等缺点, 无法 满足人们对于实时快速准确感知的要求.

随着传感器向微型化、智能化、网络化和多功能化 的方向发展，同时测量多个参数的高集成传感器需要制 造工艺和分析技术的创新.

我们 ${ }^{[15]}$ 首次展示了通过压阻柔性可穿戴电子传感 器多通道分析进行表情识别的概念(见图 8). 利用图案 化硅柱阵列模板，与含有纳米颗粒的组装液及柔性薄膜 构筑的三明治夹层, 随着溶剂的挥发, 气一液一固三相接 触线的有序收缩，从而形成了周期与振幅可控的曲线形 液桥阵列, 此不断缩小的液桥空间为纳米颗粒的沉积提 供了规则有序的组装空间，待溶剂挥发干，便在柔性薄 膜上形成了规则的微纳米级曲线阵列，真空蒸镀上金电 极, 得到对微小形变有稳定电阻变化的传感器芯片. 不 同周期和振幅比的曲线阵列传感器对应变的电阻响应 曲线有明显差异. 一组传感器贴附于被监测者的体表皮 肤, 进行数据采集与分析, 实时监测人在不同环境和心 理条件下, 体表微形变的相关生理反应, 用于识别微 笑、大笑、惊讶、悲伤、恐惧、沮丧、生气和放松八种 主要的面部表情.

\section{4 运动监测}

当代社会, 人们越来越需要对人体活动进行实时监 测. 在能与人体交互的诊疗电学设备中, 监控人体运动 的应力传感器备受瞩目. 监测人体运动的策略可以分为 两种: 一种是监测大范围运动, 例如手、胳膊和腿的弯 曲运动; 另一种是监测像呼吸, 吞咽和说话过程中胸和 颈的细微运动. 适用于这两种策略的传感器必须具备好 的拉伸性和高灵敏度. 而传统的基于金属和半导体的应 力传感器不能胜任. 所以, 具备好的拉伸性和高灵敏度 的柔性可穿戴电子传感器在运动监测领域至关重要.

Park 等 ${ }^{[60]}$ 发展了一种基于石墨烯的应力传感器, 巧 妙地运用可拉伸的纱线层层组装石墨烯纳米粒子分散 液和 PVA 溶液(见图 9). 显然, 这个过程是简便、廉价和 规模化的溶液过程. 通过不同的纱线结构组装了压阻性 质不同的应力传感器. 其中, 橡胶纱线型(RY)传感器可 以探测喉部和胸部小幅度振动, 尼龙包覆的橡胶纱线型 $(\mathrm{NCRY})$ 传感器可以探测大幅度运动. 这种传感器可以 轻易的辨别身体运动的部分.

Kim 等 ${ }^{[61]}$ 通过干纺的方法制备了高度取向性的碳 纳米管纤维弹性应力传感器. 因为其在柔性基底上制 备, 结果得到了超过 $900 \%$ 的拉紧程度, 高灵敏度, 快速 

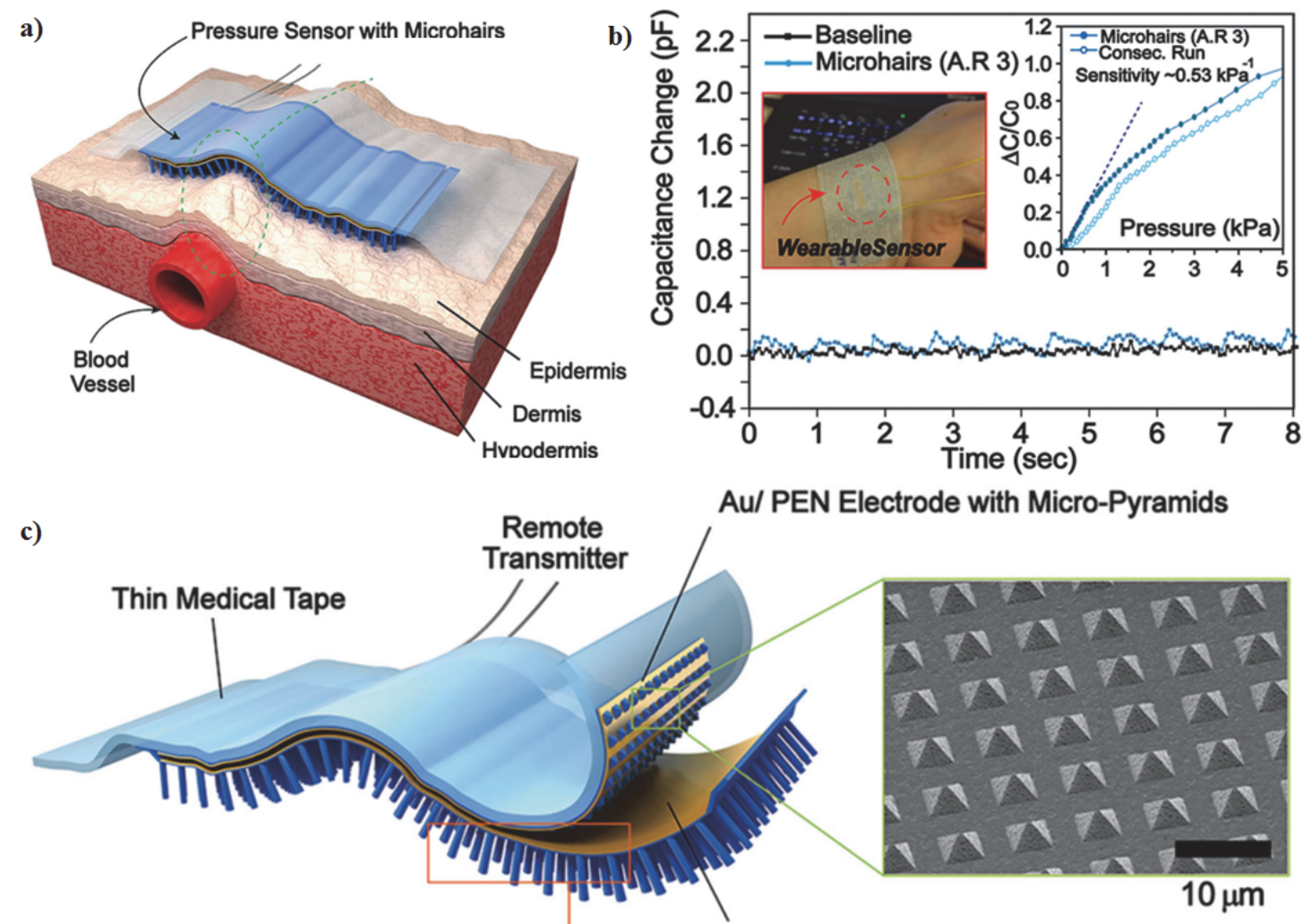

Au/ PEN Electrode with Microhair Structures

图 7 柔性电子传感器在脉搏监测上的应用

Figure 7 Applications of wearable sensors on pulse detection

(a) Schematic illustration to detect pulse on a human's neck with our microhair sensor. (b) Measure pulse waves of the radial artery. (c) Cross-sectional diagram of the microhair-structured sensor. The pyramid-shaped PDMS dielectric layer was placed between the two Au electrodes on PEN plastic substrates. The pressure sensor was subsequently added on a layer of microhair-structured biocompatible polymer(PDMS) to improve conformal contacts with skin

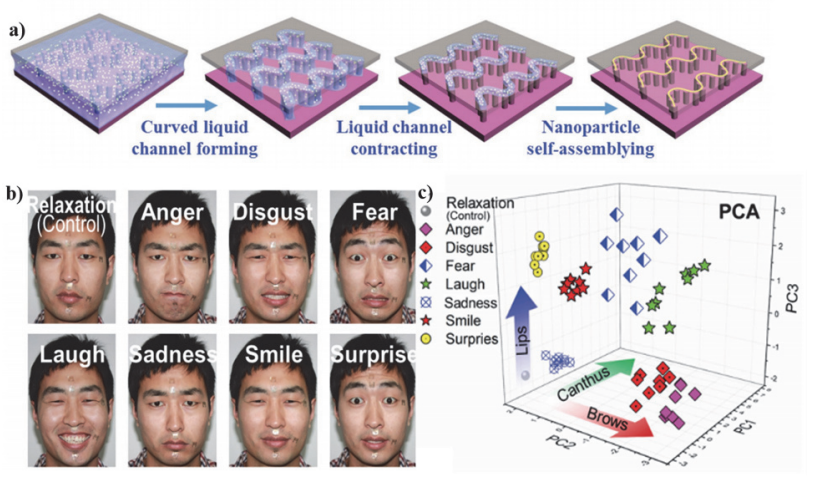

图 8 柔性可穿戴传感器在表情识别上的应用

Figure 8 Flexible expression recognition sensors

(a) The schematic illustration of the nanoparticle self-assembly process induced by pillar-patterned template. (b) The nanocurves array chips were attached at six selective positions on facial skin, which included the characteristic muscle groups. (c) 3D representation of PCA result shows a clear clustering of the eight different facial expressions as analytes

响应和好的持久性. 高弹性的应变仪在不同体系中具有 巨大应用潜力，如人体运动和可穿戴传感器.

Hata 等 ${ }^{[62]}$ 制备了定向排列的单壁碳纳米管薄膜. 当拉伸时, 碳纳米管破裂成岛一桥一间隙结构, 形变可以 达到 $280 \%$ (是传统金属拉力计的 50 倍). 将这种传感器
组装在长袜、绷带和手套上, 可以监测不同类型的动作, 比如移动、打字、呼吸和讲话等.

\section{6 总结与展望}

随着智能终端的普及, 可穿戴电子设备展现出巨大 的市场前景. 传感器作为可穿戴设备的核心部件, 将对 其未来功能发展产生重要影响. 本文综述了近年来柔性 可穿戴电子传感器方面的最新研究进展, 包括压阻、电 容、压电、力致发光和摩擦电五种信号转换机制, 金属、 无机半导体、有机和碳材料等柔性可穿戴电子的常用材 料, 柔性电子传感器的印刷制造及其在温度、脉搏、表 情识别和运动监测等方面的最新应用. 随着研究的进一 步深入以及各学科的广泛交叉, 更多柔性、拥有良好电 学性能的材料将被开发出来应用于可穿戴传感器. 制备 工艺方面，目前以光刻为代表的微加工技术，具有较高 的加工成本，复杂的工艺，且工艺对材料的巨大浪费等 弊端, 无法适应未来高性能柔性可穿戴器件的大规模、 低成本、高效、清洁制造. 最近几年发展的以印刷电子、 $3 \mathrm{D}$ 打印为代表的印刷制造技术，以其材料普适性、柔性 基材适用性、便捷的可定制性、大规模连续生产的高效 


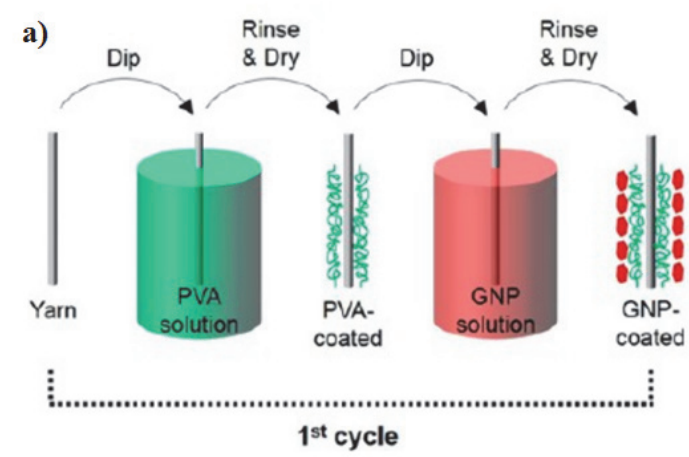

b)

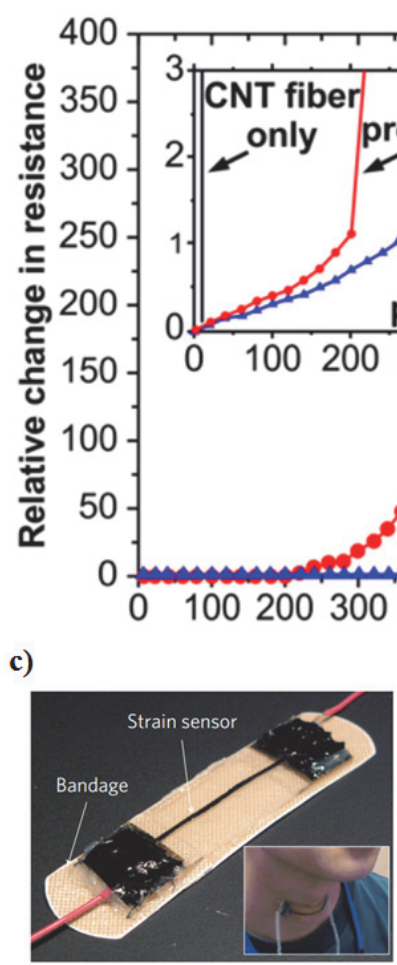

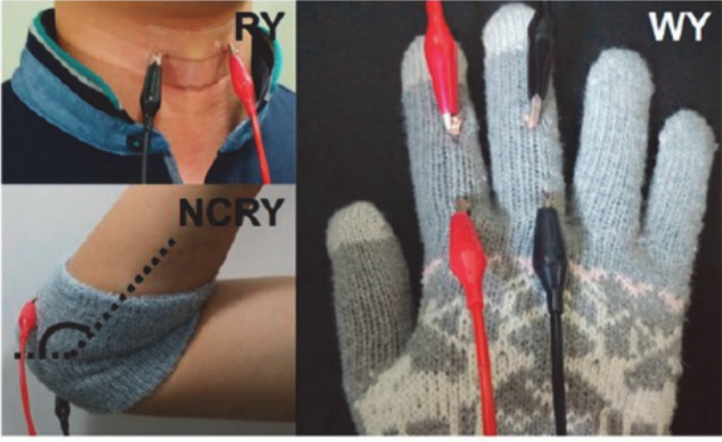

$0 \%$

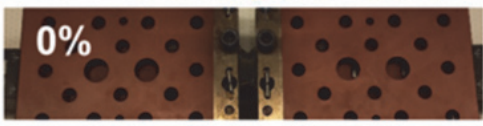

$300 \%$

$600 \%$

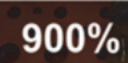

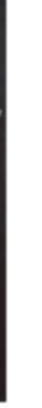

\section{.}


穿戴电子传感器将得到日益广泛的应用.

\section{作者简介}

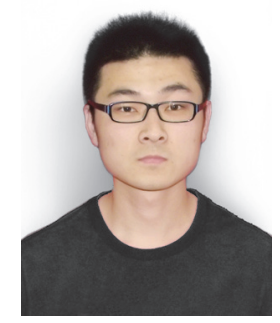

钱金金, 中国科学院化学研究所 2014 级在读直博生. 2014 年获青岛科技大学化学学士学位. 研究方向为喷, 墨打印力致 发光传感器.

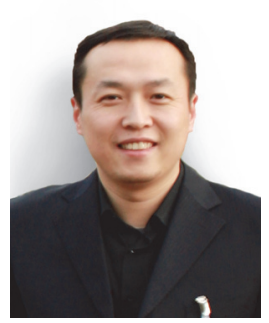

李风显, 2008 年于中国科学院化学研究所获得博士学位, 2008 2011 于美国 Bowling Green State University 从事博士 后研究; 2012 年进入中国科学院化学研究所, 任副研究员. 主 要从事高效复杂系统分析、聚合物光子晶体与纳米材料打印 功能器件研究.

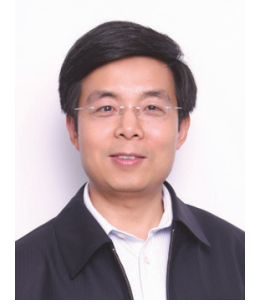

宋延林, 中国科学院化学研究所研究员、博士生导师, 绿 色印刷重点实验室主任. 主要从事信息功能材料、纳米材料与 绿色印刷技术研究.

\section{References}

[1] Maheshwari, V.; Saraf, R. Angew. Chem., Int. Ed. 2008, 47, 7808.

[2] Tee1, B. C.-K.; Chortos, A.; Berndt, A.; Nguyen, A. K.; Tom, A.; McGuire, A.; Lin, Z. C.; Tien, K.; Bae, W.-G.; Wang, H. L.; Mei, P.; Chou, H.-H.; Cui, B. X.; Deisseroth, K.; Ng, T. N.; Bao, Z. N. Science 2015, 350, 313.

[3] Velliste, M.; Perel, S.; Spalding, M. C.; Whitford, A. S.; Schwartz, A. B. Nature 2008, 453, 1098.

[4] Shintaku, H.; Nakagawa, T.; Kitagawa, D.; Tanujaya, H.; Kawano, S.; Ito, J. Sensor. Actuat. A-Phys. 2010, 158, 183.

[5] Chen, L. Y.; Tee, B. C. K.; Chortos, A. L.; Schwartz, G.; Tse, V.; Lipomi, D. J.; Wong, H. S. P.; McConnell, M. V.; Bao, Z. A. Nat. Commun. 2014, 5, 5028.

[6] (a) Park, J.; Lee, Y.; Hong, J.; Ha, M.; Jung, Y.-D.; Lim, H.; Kim, S. Y.; Ko, H. ACS Nano 2014, 8, 4689; (b) Pan, L.; Chortos, A.; Yu, G.; Wang, Y.; Isaacson, S.; Allen, R.; Shi, Y.; Dauskardt, R.; Bao, Z. N. Nat. Commun. 2014, 5, 3002.
[7] Li, R.-Z.; Hu, A.; Zhang, T.; Oakes, K. D. ACS Appl. Mater. Interfaces 2014, 6, 21721.

[8] Pan, C.; Dong, L.; Zhu, G.; Niu, S.; Yu, R.; Yang, Q.; Liu, Y.; Wang, Z. L. Nature Photon. 2013, 7, 752 .

[9] Koeppe, R.; Bartu, P.; Bauer, S.; Sariciftci, N. S. Adv. Mater. 2009, 21,3510 .

[10] Wang, S.; Lin, L.; Wang, Z. L. Nano Energy 2015, 11, 436.

[11] Choong, C.-L.; Shim, M.-B.; Lee, B.-S.; Jeon, S.; Ko, D.-S.; Kang, T.-H.; Bae, J.; Lee, S. H.; Byun, K.-E.; Im, J.; Jeong, Y. J.; Park, C. E.; Park, J.-J.; Chung, U. I. Adv. Mater. 2014, 26, 3451.

[12] Timsit, R. S. IEEE Trans. Compon. Packag. Technol. 1999, 22, 85.

[13] Gong, S.; Schwalb, W.; Wang, Y.; Chen, Y.; Tang, Y.; Si, J.; Shirinzadeh, B.; Cheng, W. Nat. Commun. 2014, 5, 3132.

[14] Zhao, J.; Wang, G. L.; Yang, R.; Lu, X. B.; Cheng, M.; He, C. L.; Xie, G. B.; Meng, J. L.; Shi, D. X.; Zhang, G. Y. ACS Nano 2015, 9, 1622 .

[15] Su, M.; F. Li, Y.; Chen, S. R.; Huang, Z. D.; Qin, M.; Li, W. B.; Zhang, X. Y.; Song, Y. L. Adv. Mater. 2016, 28, 1369.

[16] Lee, H.-K.; Chung, J.; Chang, S.-I.; Yoon, E. J. Micromech Microeng. 2011, 21, 035010 .

[17] Frutiger, A.; Muth, J. T.; Vogt, D. M.; Mengüç, Y.; Campo, A.; Valentine, A. D.; Walsh, C. J.; Lewis, J. A. Adv. Mater. 2015, 27, 2440.

[18] Lipomi, D. J.; Vosgueritchian, M.; Tee, B. C. K.; Hellstrom, S. L.; Lee, J. A.; Fox, C. H.; Bao, Z. N. Nature Nanotechnol. 2011, 6, 788.

[19] Wang, Z. L.; Song, J. Science 2006, 312, 242.

[20] Graz, I.; Kaltenbrunner, M.; Keplinger, C.; Schwodiauer, R.; Bauer, S.; Lacour, S. P.; Wagner, S. Appl. Phys. Lett. 2006, 89, 073501.

[21] Dagdeviren, C.; Su, Y.; Joe, P.; Yona, R.; Liu, Y.; Kim, Y.-S.; Huang, Y.; Damadoran, A. R.; Xia, J.; Martin, L. W.; Huang, Y.; Rogers, J. A. Nat. Commun. 2014, 5, 4496.

[22] Nour, E. S.; Sandberg, M. O.; Willander, M.; Nur, O. Nano Energy 2014, 9, 221 .

[23] Zhang, X.; Hillenbrand, J.; Sessler, G. M. J. Appl. Phys. 2007, 101, 054114.

[24] Yun, S.; Park, S.; Park, B.; Kim, Y.; Park, S. K.; Nam, S.; Kyung, K.-U. Adv. Mater. 2014, 26, 4474.

[25] Sun, Y.; Rogers, J. A. J. Mater. Chem. 2007, 17, 832.

[26] Akinwande, D.; Petrone, N.; Hone, J. Nat. Commun. 2014, 5, 5678.

[27] Lee, P.; Lee, J.; Lee, H.; Yeo, J.; Hong, S.; Nam, K. H.; Lee, D.; Lee, S. S.; Ko, S. H. Adv. Mater. 2012, 24, 3326.

[28] Sekitani, T.; Nakajima, H.; Maeda, H.; Fukushima, T.; Aida, T.; Hata, K.; Someya, T. Nature Mater. 2009, 8, 494.

[29] Brosteaux, D.; Axisa, F.; Gonzalez, M.; Vanfleteren, J. IEEE Electron Device Lett. 2007, 28, 552.

[30] Zhang, Y. H.; Wang, S. D.; Li, X. T.; Fan, J. A.; Xu, S.; Song, Y. M.; Choi, K. J.; Yeo, W. H.; Lee, W.; Nazaar, S. N.; Lu, B. W.; Yin, L.; Hwang, K. C.; Rogers, J. A.; Huang, Y. G. Adv. Funct. Mater. 2014, 24, 2028.

[31] Choi, W. M.; Song, J.; Khang, D.-Y.; Jiang, H.; Huang, Y. Y.; Rogers, J. A. Nano Lett. 2007, 7, 1655.

[32] Kim, D.-H.; Song, J.; Choi, W. M.; Kim, H.-S.; Kim, R.-H.; Liu, Z.; Huang, Y. Y.; Hwang, K.-C.; Zhang, Y.-W.; Rogers, J. A. Proc. Natl. Acad. Sci. U. S. A. 2008, 105, 18675.

[33] Fan, J. A.; Yeo, W. H.; Su, Y. W.; Hattori, Y.; Lee, W.; Jung, S. Y.; Zhang, Y. H.; Liu, Z. J.; Cheng, H. Y.; Falgout, L.; Bajema, M.; Coleman, T.; Gregoire, D.; Larsen, R. J.; Huang, Y. G.; Rogers, J. A. Nat. Commun. 2014, 5, 3266.

[34] Jiang, J. K.; Bao, B.; Li, M. Z.; Sun, J. Z.; Zhang, C.; Li, Y.; Li, F. Y.; Yao, X.; Song, Y. L. Adv. Mater. 2016, 28, 1420.

[35] Park, M.; Im, J.; Shin, M.; Min, Y.; Park, J.; Cho, H.; Park, S.; Shim, M.-B.; Jeon, S.; Chung, D.-Y.; Bae, J.; Park, J.; Jeong, U.; Kim, K. Nature Nanotechnol. 2012, 7, 803.

[36] Wang, X.; Zhang, H.; Yu, R.; Dong, L.; Peng, D.; Zhang, A.; Zhang, Y.; Liu, H.; Pan, C. F.; Wang, Z. L. Adv. Mater. 2015, 27, 2324.

[37] Park, S.; Pitner, G.; Giri, G.; Koo, J. H.; Park, K.; Kim, H.; Wang, R.; Sinclair, H. S.; Wong, P.; Bao, Z. N. Adv. Mater. 2015, 27, 2656.

[38] Zhan, X.; Facchetti, A.; Barlow, S.; Marks, T. J.; Ratner, M. A.; Wasielewski, M. R.; Marder, S. R. Adv. Mater. 2011, 23, 268.

[39] Wang, H. L.; Wei, P.; Li, Y. X.; Han, J.; Lee, H. R.; Naab, B. D.; Liu, N.; Wang, C. G.; Adijanto, E.; Tee, B. C. K.; Morishita, S.; Li, Q. C.; Gao, Y. L.; Cui, Y.; Bao, Z. N. Proc. Natl. Acad. Sci. U. S. A. 2014, $111,4776$.

[40] Someya, T.; Sekitani, T.; Iba, S.; Kato, Y.; Kawaguchi, H.; Sakurai, T. Proc. Natl. Acad. Sci. U. S. A. 2004, 101, 9966.

[41] Mannsfeld, S. C. B.; Tee, B. C. K.; Stoltenberg, R. M.; Chen, C. V. 
H. H.; Barman, S.; Muir, B. V. O.; Sokolov, A. N.; Reese, C.; Bao, Z. N. Nature Mater. 2010, 9, 859.

[42] Schwartz, G.; Tee, B. C. K.; Mei, J.; Appleton, A. L.; Kim, D. H.; Wang, H.; Bao, Z. N. Nat. Commun. 2013, 4, 1859.

[43] Zang, Y.; Zhang, F.; Huang, D.; Gao, X.; Di, C.-A.; Zhu, D. B. Nat. Commun. 2015, 6, 6269.

[44] Chun, K.-Y.; Oh, Y.; Rho, J.; Ahn, J.-H.; Kim, Y.-J.; Choi, H. R.; Baik, S. Nature Nanotechnol. 2010, 5, 853.

[45] Chae, S. H.; Yu, W. J.; Bae, J. J.; Duong, D. L.; Perello, D.; Jeong, H. Y.; Ta, Q. H.; Ly, T. H.; Vu, Q. A.; Yun, M.; Duan, X. F.; Lee, Y. H. Nature Mater. 2013, 12, 403.

[46] Bai, C. L. Chin. Sci. Bull. 2009, 54, 1941. (白春礼, 科学通报, 2009, 54, 1941.)

[47] Kuang, M. X.; Wang, J. X.; Wang, L. B.; Song, Y. L. Acta Chim. Sinica 2012, 70, 1889. (广旻睘羽, 王京霞, 王利涁, 宋延林, 化学学 报, 2012, 70, 1889.)

[48] Sun, J. Z.; Kuang, M. X.; Song, Y. L. Prog. Chem. 2015, 27, 979. (孙加振, 广旻噮棚, 宋延林, 化学进展, 2015, 27, 979.)

[49] Kuang, M. X.; Wang, L. B.; Song, Y. L. Adv. Mater. 2014, 26, 6950.

[50] Zhang, Z. L.; Zhang, X. Y.; Xin, Z. Q.; Deng, M. M.; Wen, Y. Q.; Song, Y. L. Adv. Mater. 2013, 25, 6714 .

[51] Su, B.; Zhang, C.; Chen, S. R.; Zhang, X. Y.; Chen, L. F.; Wu, Y. C.; Nie, Y. W.; Kan, X. N.; Song, Y. L.; Jiang, L. Adv. Mater. 2014, 26, 2501.

[52] Chen, S. R.; Su, M.; Zhang, C.; Gao, M.; Bao, B.; Yang, Q.; Su, B.; Song, Y. L. Adv. Mater. 2015, 27, 3928.
[53] Han, I. Y.; Kim, S. J. Sens. Actuators A 2008, 141, 52.

[54] Hattori, Y.; Falgout, L.; Lee, W.; Jung, S. Y.; Poon, E.; Lee, J. W.; Na, I.; Geisler, A.; Sadhwani, D.; Zhang, Y. H.; Su, Y. W.; Wang, X. Q.; Liu, Z. J.; Xia, J.; Cheng, H. Y.; Webb, R. C.; Bonifas, A. P.; Won, P.; Jeong, J. W.; Jang, K. I.; Song, Y. M.; Nardone, B.; Nodzenski, M.; Fan, J. A.; Huang, Y. G.; West, D. P.; Paller, A. S.; Alam, M.; Yeo, W. H.; Rogers, J. A. Adv. Healthcare Mater. 2014, 3, 1597.

[55] Hong, S. Y.; Lee, Y. H.; Park, H.; Jin, S. W.; Jeong, Y. R.; Yun, J.; You, I.; Zi, G.; Ha, J. S. Adv. Mater. DOI: 10.1002/adma. 201504659.

[56] Schwartz, G.; Tee, B. C. K.; Mei, J. G.; Appleton, A. L.; Kim, D. H.; Wang, H. L.; Bao, Z. N. Nat. Commun. 2013, 4, 1859.

[57] Pang, C. F.; Koo, J. H.; Nguyen, A.; Caves, J. M.; Kim, M.; Chortos, A.; Kim, K.; Wang, P. J.; Tok, J. B.-H.; Bao, Z. N. Adv. Mater. 2015, $27,634$.

[58] Belanger, M. C.; Marois, Y. J. Biomed. Mater. Res. 2001, 58, 467.

[59] Shan, C. F.; Gong, S. G.; McOwan, P. W. Image Vision Comput. 2009, 27, 803 .

[60] Park, J. J.; Hyun, W. J.; Mun, S. C.; Park, Y. T.; Park, O. O. ACS Appl. Mat. Interfaces 2015, 7, 6317.

[61] Ryu, S.; Lee, P.; Chou, J. B.; Xu, R.; Zhao, R.; Hart, A. J.; Kim, S.-G. ACS Nano 2015, 9, 5929.

[62] Yamada, T.; Hayamizu, Y.; Yamamoto, Y.; Yomogida, Y.; Izadi-Najafabadi, A.; Futaba, D. N.; Hata, K. Nature Nanotechnol. 2011, 6, 296. 